\title{
O FINANCIAMENTO DOS SISTEMAS DE SAÚDE DO BRASIL E PORTUGAL: QUAL O FUTURO?
}

\author{
BRAZIL AND PORTUGAL HEALTH SYSTEMS' FUNDING: WHAT IS THE \\ FUTURE?
}

\section{Janice Dornelles de Castro}

Doutora em Saúde Coletiva pela Universidade Estadual de Campinas. Professora Adjunta da Universidade Federal do Rio Grande do Sul Faculdade de Ciências Econômicas - Programa de Pós-Graduação em Economia.

E-mail: janice.castro@ufrgs.br

\section{Soraya Vargas Cortes}

Doutora em Social Policy And Administration pela London School Of Economics And Political Science. Professora Associada da Universidade Federal do Rio Grande do Sul - Instituto de Filosofia e Ciências Humanas - Programa de Pós-Graduação em Sociologia. E-mail: vargascortes@ufrgs.br

\section{Resumo}

O artigo examina as diferentes alternativas de financiamento dos sistemas de saúde pública no Brasil e Portugal, identificando o processo de organização, as modalidades de financiamento e o papel dos diferentes atores. A caracterização detalhada do financiamento, idéias, crenças, interesses das partes, arranjos institucionais, mobilização de comunidades profissionais, coligações e poder de decisão foram realizados. As informações foram analisadas à luz do legado institucional e do contexto político macro. O objetivo foi identificar os processos endógenos e peculiares em cada país e as características comuns relacionados com a ação de atores e processos institucionais supranacionais em relação à discussão de como as políticas de financiamento são definidas.

Palavras-chave: Sistemas de saúde; Financiamento da Assistência à Saúde; Saúde pública. 


\section{Abstract}

We analyzed different funding alternatives of Brazil and Portugal Public Health Systems, pointing out organization process, funding strategies and the role of different actors. The detailed funding description, ideas, beliefs, stakeholders interests, institutional arrangements, professional communities mobilization, coalitions and decision-making authority was performed. These data were analyzed in the focus of institutional legacy and macro political. The objective was to identify the endogenous and peculiar processes in each country and the matching features according to the action of actors and supranational institutional processes related to the discussion of how funding policies are defined.

Keywords: Health Systems; Healthcare Funding; Public Health.

\section{Introdução}

O problema fundamental da investigação indaga sobre o papel das comunidades de políticas, articulando atores societais e burocracias governamentais, na definição da agenda política governamental, isso, sem desconsiderar a importância das instituições políticas e econômicas que modelam a ação desses atores. 0 objeto empírico da pesquisa é a análise de como se estruturaram as modalidades de financiamento do sistema público de saúde, no Brasil e em Portugal, enfatizando o papel dos atores envolvidos na sua estruturação e dessas modalidades de financiamento no processo de desenvolvimento econômico dos países.

No pós segunda guerra, os diferentes Estados de bem-estar social implantados nos países da Organização para a Cooperação e Desenvolvimento Econômico (OCDE) contribuíram para a redução da pobreza e melhoraram a saúde da população. No final dos anos 70, esses modelos se tornaram mais individualistas, fenômeno que promoveu, em países de baixa e média renda, um problema com dívidas, empréstimos e financiamentos com países de alta renda e instituições financeiras, criando muitas dificuldades para o estabelecimento de um sistema de saúde igualitário. ${ }^{1}$ Durante um longo período a discussão sobre o desenvolvimento econômico e saúde perde força, sendo subordinada ao discurso e teses neoliberais hegemônicos que priorizavam a discussão da reorganização macroeconômica dos países, do papel do Estado ou Estado mínimo, reorientação do gasto público, da modernização da gestão e das políticas de privatização. $2,3,4,5,6$

Apenas nos anos 2000 retoma-se a discussão do desenvolvimento econômico e saúde. Para Mollo "O interesse crescente pelo tema desenvolvimentismo, após três décadas de arquivamento acadêmico, tendo em vista a hegemonia ortodoxa neoliberal, é alvissareiro."7:23 Nesse período muitas reformas nos sistemas de saúde foram propostas e eventualmente implementadas visando a redução do papel do Estado.

De acordo com Jomo ${ }^{8}$ que realizou um estudo sobre os efeitos da globalização e da liberalização da economia em cinco países asiáticos: Indonésia, Malásia, Coréia, Tailândiae Taiwan, oConsensode Washington e as propostas do Banco Mundial criaram uma hegemonia do pensamento econômico em relação à eficiência das políticas de minimização do Estado para promover o crescimento econômico. As intervenções seletivas do Estado, em que pese tenham contribuído para um crescimento rápido, mudanças estruturais, desenvolvimento da capacidade de competição internacional da indústria, aumento do emprego e dos salários médios, não teve consequências 
diretas na diminuição da desigualdade ou numa melhor distribuição de renda. $O$ pequeno investimento na criação de redes de proteção social, para se contrapor as rápidas mudanças estruturais e aos ciclos econômicos promoveu um desastre social quando da crise monetária e financeira de 1997 e a recessão que se seguiu, evidenciando inadequação dessa política.

Os Objetivos do Milênio para 2015 propostos pela ONU considera como requisito para o desenvolvimento econômico e social o investimento em saúde, ciência e tecnologia, de acordo com Morel $^{9}$ esse deve ser o grande desafio para os países em desenvolvimento, pois será necessário organizar sistemas de pesquisas em saúde baseados em prioridades sanitárias e ainda, assegurar a incorporação dos resultados na política e ações de saúde. Para Gadelha ${ }^{10}$ todos os países que se desenvolveram e passaram a competir em melhores condições com os mais avançados, associaram uma indústria forte com uma base endógena de conhecimento, de aprendizado e de inovação. Utilizando dados de comércio exterior, demonstram como a desconsideração da lógica do desenvolvimento nas políticas de saúde levou a uma situação de vulnerabilidade econômica do setor que pode limitar os objetivos de universalidade, equidade e integralidade. Nesse contexto, um país que pretende chegar a uma condição de desenvolvimento e de independência requer, ao mesmo tempo, indústrias fortes e inovadoras e um sistema de saúde inclusivo e universal.

Após um período de poucas discussões, o atual momento é de muitas propostas em relação ao conceito de desenvolvimento econômico, saúde e a relação com a desigualdade. A Comissão de Determinantes Sociais da Saúde da OMS ${ }^{11}$ propôs uma nova visão para o desenvolvimento econômico, sugere que saúde e equidade em saúde devem ser o principal resultado das políticas sociais e, em que pese a importância do crescimento econômico, especialmente para os países pobres, que não é suficiente para garantir equidade nos resultados das políticas sociais, propondo um modelo para explicar o que determina as desigualdades em saúde.

Em relação a esta discussão temos a posição de Mirvis ${ }^{12}$ em artigo que trata dos motivos pelos quais a melhora na saúde gera desenvolvimento econômico, focando a relação entre a saúde da população e a sua produtividade econômica. Para comprovar esta relação são feitas análises de períodos da história econômica, ficando evidente que vários dos booms econômicos estão associados com controle de doenças e melhora na nutrição. Neste caso, saúde é uma commodity macroeconômica e a infraestrutura do sistema de saúde de países em desenvolvimento é um pré-requisito para estimular o desenvolvimento econômico, assim como a boa saúde da população é de vital importância para a redução da pobreza e para o crescimento econômico de curto e longo prazo. Acredita que a produtividade econômica está diretamente relacionada com a saúde por quatro fatores principais: a) Doenças reduzem o bem-estar econômico dos indivíduos pela redução de renda familiar, gasto com cuidados de saúde, perdas de rendas futuras, redução de expectativa de vida e diminuição da produtividade devido ao estado físico e psicológico do indivíduo; b) Falta de saúde têm consequências no futuro, pois doenças durante a gestação ou na primeira infância têm efeitos nas habilidades cognitivas e psicológica, podendo limitar a educação. Além disso, a doença de uma geração pode influenciar diretamente na outra, pais doentes não oferecem apoio necessário para a educação; c) Altas taxas de mortalidade infantil e de fertilidade levam famílias mais pobres a compensar a alta taxa de mortalidade, tendo um maior número de filhos, restando pouca renda para ser gasta 
por filho; d)Falta de saúde em uma área tem consequências para toda a sociedade, pois reduz os investimentos externos, o turismo, afeta a força de trabalho e mobiliza os escassos recursos, deixando outras esferas sem cobertura.

Discute ainda que o valor econômico do aumento na expectativa de vida é tão grande quanto o valor de todos os outros bens de consumo e serviços juntos. Cada ano de vida perdido devido a morte prematura, leva a uma perda econômica equivalente a três vezes a renda anual desse indivíduo e o aumento de $10 \%$ na expectativa de vida está associado com um crescimento econômico de 0,3 a $0,4 \%$ por ano. ${ }^{12}$ Podemos concluir que existe uma forte relação entre saúde e desenvolvimento econômico, mas como garantir a diminuição das desigualdades relacionadas com a saúde e obter um desenvolvimento econômico que seja socialmente justo?

Com objetivo de esclarecer essa questão inúmeros autores discutem a diferença entre desigualdade em saúde e desigualdade social em saúde. A desigualdade em saúde é a ausência de diferenças em saúde, injustas e inevitáveis entre grupos populacionais definidos social, demográfica e geograficamente. As desigualdades sociais em saúde, por sua vez, referem-se as distintas oportunidades e recursos relacionados com a saúde que as pessoas acessam em função de sua classe social, sexo ou etnia que tendem a aumentar, pois a saúde melhora mais rápido para os indivíduos de classes sociais mais elevadas, entretanto, essa tendência poderá ser alterada se forem aplicadas as intervenções e políticas públicas sanitárias e sociais adequadas. ${ }^{13,14,15}$

Segundo Fleurbaey eSchokkarert ${ }^{14}$ num mesmo grupo social existem diferenças em saúde que podem ser completamente identificadas por diferença nos costumes, como o fumo, por exemplo, essas desigualdades são justas. As diferenças no uso dos serviços de saúde que se refletem na diferença de necessidades, por sua vez, são desejáveis. Existem desigualdades legítimas e não-legítimas, sendo legítimas (justas) as que dependem da responsabilidade individual. Por isso, as diferentes visões sobre equidade são apenas questão do que a pessoa considera como legítimo ou ilegítimo.

Ainda em relação à discussão da necessidade da construção de um desenvolvimento econômico socialmente justo, Borrelle Artazcoz ${ }^{13}$ apresentam um modelo para avaliar desigualdades que foi proposto pela Comissão de Determinantes Sociais da Saúde da Organização Mundial da Saúde (OMS) como sendo composto por um conjunto de determinantes: a) os estruturais, relativos ao contexto socioeconômico e posição socioeconômica e b) intermediários, relativo as circunstâncias materiais, psicossociais e fatores comportamentais e biológicos. ${ }^{13}$ Blas, ${ }^{1}$ por sua vez, apresenta outra proposta da Comissão de Determinantes Sociais da Saúde da OMS sobre os papéis que o Estado e a sociedade devem desempenhar para gerar maior equidade em saúde segundo nove redes de conhecimento (nine knowledge networks) são elas: desenvolvimento da primeira infância, condições de emprego, sistemas de saúde, contexto urbano, globalização, exclusão social, igualdade de gêneros, condições de saúde pública prioritárias, mensuração e evidências. Ou seja, os governos podem agir como: a) provedor dos direitos humanos e serviços essenciais; b)facilitador de políticas que forneçam bases para o desenvolvimento da equidade em saúde;c) coletor e monitor de dados sobre sua população que forneçam informações sobre equidade em saúde. A sociedade tem um papel fundamental para a redução da desigualdade. ${ }^{1} \mathrm{O}$ engajamento das comunidades nas decisões é mais apropriado e efetivo, indicando o que a 
população prefere e precisa. Para que esses grupos da sociedade funcionem melhorando o sistema,é necessário que o governo reconheça a legitimidade política da sociedade civil e a voz da comunidade, designando políticas que Ihes deem real poder.

Para Fleurbaey e Schokkarert ${ }^{14}$ a igualdade no uso dos recursos de cuidados em saúde não determina a igualdade nos cuidados de saúde, pois o que realmente importa são os impactos desses cuidados de saúde.Por isso, a preocupação deve ser sobre a composição do consumo em saúde, mesmo que o uso dos recursos seja igualitário. Se uma pessoa adquire tratamento de qualidade inferior, devido a fatores que não são de sua responsabilidade, é difícil sustentar que não há injusta desigualdade em saúde. Porém, remover as desigualdades em cuidados de saúde não é suficiente para remover completamente as desigualdades em saúde.

Como vimos anteriormente, a busca pela equidade é um processo cujo primeiro passo é o reconhecimento e detalhamento dasdesigualdadessociais. ${ }^{1,12,13,14,16,17}$ Portanto é muito importante para o planejamento das políticas públicas, a construção de um índice de condições de vida e saúde (ICVD) que refletisse a complexidade da realidade de saúde e doença. Luiz ${ }^{18}$ elaborou esse índice, utilizando quatro dimensões: econômica, consciência e conduta, ecológica e biológica e, com base em 16 indicadores: demografia, renda, oferta e produção de serviços de saúde, meio ambiente e habitação, educação. ${ }^{18}$

Este é um estudo que integra discussões da economia e da sociologia política, pois trata das relações entre Estado e sociedade, focalizando os fatores e os atores que ocasionam a mudança no curso das ações governamentais provocando a interrupção da vigência de padrões de funcionamento de políticas já existentes, ao ponto de promoverem rupturas ou rearranjos incrementais e deflagrarem inovações político-institucionais.

Tem como propósito investigar o processo de construção de políticas públicas na área da saúde, no caso o financiamento dos sistemas públicos de saúde, em uma perspectiva comparada, abordando, por um lado, o modo como atores societais e estatais agem na defesa de propostas que vêm a ocupar lugar central no debate público setorialespecializado. Tantono Brasil, quanto em Portugal, o modo como se estruturaram as políticas de financiamento dos sistemas públicos cumpriram com o papel de criar e sustentar um setor dinâmico de acumulação capital ${ }^{19}$ e, ao mesmo tempo, organizar o mercado de trabalho para o capital, onde os atores sociais visam "exercer influência por meio da participação em associações e movimentos democráticos"20:427 e os atores de mercado, controlar, administrar a produção e maximizar seus lucros e os decisores econômicos têm a produtividade e o lucro como o sentido principal de suas ações políticas, sendo guiados por tais imperativos. Importante salientar que as reformas ocorridas nos sistemas de saúde dos dois países foram concomitantes com importantes mudanças políticas e demográficas, como o final dos períodos ditatoriais, a integração em blocos econômicos (Comunidade Europeia e Mercosul), a urbanização, drástica diminuição da mortalidade infantil e aumento da esperança da vida. ${ }^{21}$

\section{Objetivos}

Analisar os processos de formulação das políticas de financiamento dos sistemas públicos de saúde e a sua inclusãona agenda política governamental. 


\section{Métodos}

Este é um estudo empírico que usa a pesquisa bibliográfica e documental e dados secundários do tipo séries temporais. ${ }^{22}$

Inicialmente foi feita a revisão de literatura sobre financiamento dos sistemas públicos de saúde no Brasil e Portugal. Essa revisão foi realizada com base nos principais artigos e livros publicados sobre o tema, utilizando as palavras-chave financiamento da saúde, economia da saúde, políticas de financiamento da saúde e gestão em saúde. A primeira seleção foi a partir da indicação de artigos ou livros considerados relevantes pelos entrevistados, uma segunda seleção foi usando as mesmas palavras-chaveno Portal de Periódicos da Coordenação de Aperfeiçoamento de Pessoal de Nível Superior (Capes), no períodode 1980a 2012. E por fim, o mesmo levantamento foi realizado no acervo da biblioteca da Escola Nacional de Saúde, em Lisboa, Portugal.

A pesquisa documental foi realizada com base principalmente nos documentos produzidos por atores individuais e coletivos, estatais e societais analisando suas posições em relação às propostas de políticas de financiamento, produzido no mesmo período. $A$ análise da literatura e o exame de documentos, serviram como apoio para que os pesquisadores realizassem interpretações sobre o processo sócio-político de construção das agendas governamentais referentes ao financiamento público dos sistemasde saúde no Brasil e Portugal.

A segunda etapa foi de coleta e sistematização de dados de financiamento, realizada através de visitas aos organismos de gestão financeira do Serviço Nacional de Saúde português;coleta e análise de documentos, dados secundários sobre financiamento e gasto público e coleta de informações nos sistemas de informação
doMinistério da Saúde no Brasil. Após a coleta os dados foram tabulados para a análise e comparação das informações referentes ao Brasil e osobtidos em Portugal.

\section{Discussão e Resultados}

A análise sistemática da literatura, o exame de documentos, permitiu chegarmos a interpretações que, ancoradas no debate da literatura, permitiram avançar o conhecimento sobre o processo sociopolítico de construção das agendas governamentais referentes ao financiamento público dos sistemas de saúde no Brasil e Portugal, identificando os processos endógenos e peculiares a cada país e de características comuns relacionadas àsaçõesde atores e aos processos institucionais supranacionais.

A seguir apresentamos a discussão sobre a evolução do financiamento dos sistemas de saúde dos países estudadosdesde a década de 1970 até os anos 2000. Ambos os países propunham reformas amplas e ambiciosas ${ }^{23}$ que tinham como principaisobjetivos a unificação dos sistemas (públicos, previdenciários e privados) com coordenação das ações, universalidade e integralidade do acesso, descentralização e ampliação da oferta pública, ou seja, das atribuições do Estado. Alguns dos objetivos foram alcançados, mas o processo ocorreu com avanços e recuos. ${ }^{21,24}$

No Brasil, a saúde como direito e como dever do Estado surgiu nos anos $30 \mathrm{com} o$ modelo nacional-desenvolvimentista, nas décadas seguintes a hegemonia das políticas de cunho liberal afetou a organização dos serviços de saúde. Atualmente a Nova Escola Desenvolvimentista, propõe modelos econômicos dinâmicos, politicamente democráticos e socialmente inclusivos. ${ }^{17}$ Os serviços de saúde no Brasil tiveram e 
tem um papel relevante na organização da rede urbana e delimitação territoriais. A política de descentralização e regionalização do Sistema Único de Saúde (SUS) com os correspondentes investimentos federais, inserem a saúde no novo modelo de desenvolvimento brasileiro, historicamente marcado por desigualdades econômicas,sociais e territoriais. ${ }^{16}$

\section{Década de 1970}

Em Portugal o regime autoritário prolongou-seaté 1974, nesse período houve a expansão da cobertura através da assistência hospitalar e ambulatorial, grandes mudanças ocorreram em $1976 \mathrm{com}$ o artigo 64 da Constituição Federal que criou o Serviço Nacional de Saúde - SNI "universal, geral e gratuito" cuja orientação da ação deve ser do Estado, a aprovação de legislação complementar não ocorreu imediatamente. Nessa época coexistiam três subsistemas: Saúde Pública, Previdenciário e Privado. ${ }^{24}$

A Saúde Pública garantia formalmente a cobertura para toda a população, a provisão de serviços é exclusivamente públicacom financiamento também público, a oferta de serviços é em todos os níveis de complexidade, mas limitada e existem dificuldades de acesso.O sistema Previdenciário é centralizado num instituto federal e cobre aos trabalhadores que financiam seu funcionamento com as contribuições sobre a folha de salários. A provisão dos serviços é pública e privada com expansão maior dos serviços privados em todos os níveis de complexidade.O sistema privado é residual nessa época, composto pelos seguros privados, pagamento direto e as instituições de caridade, édescentralizado e o atendimento em todos os níveis de complexidade é feito mediante pagamento.
O regime político do Brasil nessa década era autoritário e a proposta de política para a saúde era de expansão dos serviços através do regime previdenciáriocom financiamento eprovisão privada de serviços e da saúde pública, especialmente focada na atenção primária, com financiamento público. Assim como em Portugal, também subsistiam três subsistemas: saúde pública, previdenciário e privado.A saúde pública era composta pelo Ministério da Saúde e pelas secretarias estaduais e municipais de saúde que formalmente garantiam a cobertura de toda a população, no entantode fato, atendiam as populações pobres dos centros urbanos. O financiamento e a provisão de serviços eram públicos com recursos gerais do orçamento. ${ }^{21,23}$

O sistema previdenciário era centralizado numa instituição federal, financiada com a contribuição dos trabalhadorese recursos públicos, a provisão de serviços era principalmente privada para todos os níveis de complexidade.O sistema privado também composto pelos seguros privados instituições de caridade e pagamento direto, era descentralizado e $o$ atendimento dependia do pagamento direto ou de seguro. $\mathrm{O}$ atendimentoera feito em todos os níveis de complexidade. ${ }^{21,23}$

Os dois países possuem característica similares nesse período, tendo os regimes autoritários forte influência na definição das políticas de saúde que influenciavam a forma de estruturação dos sistemas de saúde fragmentados em subsistemas, tendo como principal provedor de serviçoso setor privado e apresentando sériasdificuldades de acesso aos serviços para a população, especialmente longe dos centros urbanos. ${ }^{23}$ 


\section{Década de 1980}

Nesse período a discussão das ideias neoliberais ganha força no mundo e influenciando as propostas de Reforma do Estado, buscando a diminuição de suas funções e um "novo gerencialismo" público é aplicado à área da saúde.

Portugal avança nas discussões democráticas e passa a integrar a Comunidade Econômica Europeia, em 1986. Não ocorrem grandes modificações na área da saúde em relação à existência dos três subsistemas, e permanece a indefinição da legislação complementar do SNI. A revisão Constitucional de 1989, altera o artigo 64 flexibilizando o princípio de gratuidade do sistema ao incluir a expressãotendencialmente gratuito." ${ }^{24}$

O Brasil, por sua vez, passa pelo processo de abertura democrática que se iniciaem 1985. No ano de1989 é eleitoo presidente, Fernando Collor de Melo, que inicia inúmeras reformas de cunho neoliberal no país. A proposta é da extensão da cobertura de maior complexidade, através do sistema previdenciário com provisão, principalmente privadae financiado com contribuições de trabalhadores e recursos públicos. Aexpansão da cobertura da atenção primária, viria através da saúde pública com provisão e financiamento públicos. No entanto, a promulgação da Constituição Federal de 1988 cria o Sistema Único de Saúde-SUS que unifica os sistemas previdenciário e público, gerando uma grande mudança na área da saúde no país. A proposta do SUS é usar o planejamento para organizar e distribuir os serviços conforme a necessidade da população. Estes serviços são gratuitos, o atendimento é universal e organizado regionalmente. Os serviços são hierarquizados conforme a sua complexidade tecnológica. A rede básica é a porta de entrada do sistema e deve garantir o atendimento ambulatorial básico e o desenvolvimento das ações de saúde pública. Neste modelo, a saúde éconsiderada um direito do cidadão. O processo de descentralização da saúde previsto quando da criação do SUS tomou maior impulso a partir da promulgação da Constituição Federal em 1988 e da consequente aprovação das leis 8.080 e 8.142 de 1990, as quais preveem, entre outras coisas, a descentralização e o repasse de recursos financeiros para estados e municípios, ${ }^{25,26}$ que foi realizado através da contínua absorção de novas e caras tecnologias, que trouxeram a discussão sobre custos da atenção para a ordem do dia. ${ }^{27,28,29} \mathrm{O}$ impacto desse processo se refletiu em grandes investimentos em infraestrutura assistencial (obras e equipamentos) que tinham como característica ser marcadamente desiguais entre as regiões, favorecer a criação de serviços privados através de financiamento público e organizar um sistema de saúde mais focado na oferta de serviços que na demanda. O Estado brasileiro tem sido um grande financiador das políticas de saúde desde a formação do país. A reforma do sistema proposta pela criação do SUS foi bem sucedida em diversos aspectos, no entanto, permanecem algumas questões fundamentais a serem respondidas, tendo por exemplo, como será possível garantir a equidade no acesso aos serviços de saúde com recursos financeiros limitados? Esses recursos limitados estão sendo usados para diminuir as desigualdades?

Nessa década podemos observar que a trajetória de Portugal e Brasil se distancia em termos de políticas de saúde, poismesmo que a agenda internacional neoliberal tenha atingido aos dois países, parece que seu efeito nessa décadafoi maior no Brasil ampliando tanto o subsetor privado, como o número e volume de recursos públicos destinados a financiar os provedores privadosque atendem o sistema público. ${ }^{21} \mathrm{O}$ sistema de saúde brasileiro carregaessas contradições ao longo de 
seu desenvolvimento, de um sistema para todos, em todos os níveis de complexidade, financiado com recursos públicos, cujos principais provedores de serviços são privados, consequência da fragilidade macroeconômica brasileira e dependência das agências internacionais. ${ }^{30}$

\section{Década de 1990}

Esse é o período de consolidação dos ideais e políticas neoliberais nos dois países, assim como da consolidação dos regimes democráticos.

Em Portugal, apesar da agenda neoliberal, o Estado realiza esforços para coordenar e implementar as ações de planejamento, financiamento, organização, prestação e avaliação dos cuidados de saúde. Nessa década foram criadas as taxas moderadoras, através da Portaria 338 e aprovado o Estatuto do Serviço Nacional de Saúde. Permanecem os três subsistemas, mas com algumas alterações como a cobrança de taxas moderadoras no subsistema público (copagamento), no subsistema previdenciário aumenta a provisão privada de serviços de maior complexidade e no subsetor privado cresce o número de pessoas com seguros que passa ser de $5 \%$ da população. ${ }^{23}$

No Brasil essa é uma década de grandes mudanças, com o impeachment do presidente Fernando Collor e mudança da orientação da política nacional para um ideário menos neoliberal, representado pelos presidentes Itamar Franco e Fernando Henrique Cardoso. Esse é o período em que foi criada a legislação que regulamentou o SUS (Lei 8080, Normas operacionais, etc.) e o período em que o sistema de saúde começou a se organizar de acordo com o proposto na Constituição Federal.Foi concluído o processo de unificação dos subsetores previdenciário e de saúde pública, iniciouse o processo de descentralização das ações para o nível local com a municipalização da gestão, ocorreu também a descentralização de recursos financeiro da Federação para estados e municípios, a principal fonte de recursos permanece sendo federal (70\% do gasto público com saúde). Ocorre a ampliação da cobertura gratuita em todos os níveis de complexidade, ainda com graves problemas de acesso nos níveis secundários de atenção. A provisão dos serviços é pública e privada sendo que o setor público é responsável principalmente pelas ações de atenção primaria e de alta complexidade, que são pouco lucrativas e desinteressantes para o setor privado.Nesse período ampliase para $18 \%$ a cobertura da população com seguros privados de saúde. ${ }^{21,31,32}$

\section{Década de 2000}

Os regimes democráticos estão definitivamente consolidados nos dois países e os sistemas de saúde de cada um delesapresentam características específicas. Portugal aprovou legislação que permite a "empresarialização" de hospitais e centros de saúde públicos e cria novos serviços privados. O Serviço Nacional de Saúde(SNS) formalmente atende toda a população, o financiamento é público, mas com a existência de formas de copagamento, a provisão que antes era apenas pública pode ser também privada. O subsistema previdenciário não apresenta grandes modificações e o subsistema privado se amplia, especialmente através dos seguros que cobrem $19 \%$ da população. ${ }^{23,24}$

No Brasil, o SUS consolida-se, mas com características diferentes daquelas previstas na Constituição Federal, cobre formalmente toda a população, em todos os níveis de complexidade, mas é um sistema dual em 
relação à provisão dos serviços(público privado), o financiamento é público, sem cobranças de taxas moderadoras e descentralizadas, mas a Federação reduziu sua participação no financiamento em cerca de $40 \%$ do gasto público com saúde, aumentando a responsabilidade de municípios e estados. O subsetor privado mantém sua tendência ao crescimento e cobre cerca de $23 \%$ da população. ${ }^{23}$

\section{Considerações finais}

Os sistemas de saúde dos dois países passaram por algumas etapas similares, sendo a origem dos sistemas de saúde a previdência social, com o financiamento feito através de contribuições compulsórias de empregadores e trabalhadores que tiveram importância para o desenvolvimento econômico desses países, a estrutura dos governos difere, enquanto Portugal é unitário, o Brasil é uma federação, e as unidades subnacionais possuem uma grande influência nas políticas de saúde. Ambos passaram por diversas reformas influenciadas pelas propostas da agenda internacional neoliberal e pelo momento político interno de maior ou menor democracia.

Atualmente são sistemas universais, financiados por taxação geral, no entanto, coexistem os subsistemas públicos, privados e de seguros, resultando em sistemas fragmentados. As atribuições do estado, como proposta originariamente modificouse ao longo dos anos e das diversas reformas, tendo ampliado a atribuição privada em diferentes graus.A intensidade da privatização e descentralização dos sistemas é diferenciada.

Segundo informações do Word Bank ${ }^{33}$, em dólares correntes, o gasto per capita em saúde para Portugal foi de U\$ $2.382 \mathrm{em}$ 2009 e de U\$ 1.905 em 2012, ocorrendo uma redução, enquanto para o Brasil foi de U\$ 733 em 2009 e de U\$1056 em 2012. O gasto total em relação ao PIB no período de 2009 -12 cresceu no Brasil, passando de $8,8 \%$ para $9,3 \%$ e diminuiu em Portugal de $10,8 \%$ para $9,4 \%$. O gasto público em relação ao gasto total, cresce no Brasil de $43,6 \%$ para $46,4 \%$ e diminui em Portugal de $66,5 \%$ para $62,6 \%$. Os indicadores de saúde e qualidade de vida melhoraram e em ambos é grande a preocupação com a eficiência do gasto público.

\section{Referências}

1. Blas E. Adressing social determinants of health inequities: what can state and civil society do? The Lancet.2008;372:1684-89.

2. Fleury S. Reforma Sanitária Brasileira: dilemas entre o instituinte e o instituído. CiêncSaúde Coletiva. 2009; 14(3): 743-753.

3. Macieira D. Actores y reformas ensaludenAmerica Latina. Buenos Aires:Banco Interamericano de Desarrollo; 2007. Nota Técnica de Saúde 1.

4. Meniccucci TMG. Implementação da Reforma Sanitária: formação de uma política. Saúde Soc. 2006;15(2):72-87. 


\section{Artigo Original}

5. Santos NR. Política pública de saúde no Brasil: encruzilhada, buscas e escolhas de rumos. Ciência Saúde Coletiva. 2008; 13(sup 2):2009-2018.

${ }^{6}$. Gallo E et al. Saúde Desenvolvimento e Globalização. Saúde Debate. 2005 29(71).

7. Mollo MLR, Fonseca PCD. Desenvolvimento e novo desenvolvimentismo: raízes teóricas e precisões conceituais. RevEcon Pol.2013 Abr/Jun 33(2): 222-239.

8. Jomo KS. Globalization, Liberalization and Equitable Development: Lessons from East Asia. Genebra: Unrisd; 2003.Paper No: 3: 35.

9. Morel CA pesquisa em saúde e os objetivos do milênio: desafios e oportunidades globais, soluções e políticas nacionais. Ciênc Saúde Coletiva. 2004 9(2): 261.

10. Gadelha CAG. Desenvolvimento, complexo industrial da saúde e política industrial. Rev SaúdePública. 2006 40: 11-23.

11. Who. Final Report Commissionon the Social Determinants of Health: Closing the gap in a generation. Genebra. WHO; 2008.

12. Mirvis DM, Chang, C. The relationship between health and development: health as an economic engine. J Health Human Serv Adm. 2008 31(1):30-57.

${ }^{13}$. Borrell C, Artazcoz L. Las Politicas para disminuirlas desigualdades ensalud. GacSanit. 2008 22(5):446-73.

14. Fleurbaey M,Schokkarert E. Unfair inequalities in health and health care. E. J Health Econ. 2009 28: 73-90.

15. Marmot M. Achieving health equity: from root causes to fair outcomes. The Lancet. 2007 370.

16. Gadelha CAG, et al. Saúde e territorialização na perspectiva do desenvolvimento. Ciênc Saúde Coletiva. 2011junho 16(6): 3003-3016.

17. Viana ALD, Elias PEM. Saúde e Desenvolvimento. Ciên saúde coletiva. 200712 (Sup): 17651777.

18. Luiz OC, et al. Diferenciais intermunicipais de condições de vida e saúde: construção de um indicador composto. Rev. Saúde Pública. 2009 34(1):115-22.

19. Offe C. Problemas Estruturais do Estado Capitalista. Rio de Janeiro: Tempo Brasileiro; 1984.

20. Cohen JL. Sociedade civil e globalização: repensando categorias: repensando categorias. Dados: Rev Ciências Sociais. 2003 3(46):419-459.

21. PaimJ, et al. O sistema de saúde brasileiro: a historia, avanços e desafios. The Lancet [Internet]. 2011 [cited 2011 maio 9] 11-31. Available from: www.thlancet.com.

22. Bêrni DA. Técnicas de pesquisa em economia. São Paulo: Saraiva; 2002.

23. Cortes SMV. Relatório de Pesquisa. Sociedade e Políticas Públicas: construção da agenda governamental na área de saúde. Porto Alegre: UFRGS; 2013. 


\section{Artigo Original}

${ }^{24}$. Simões J, et al. A sustentabilidade Financeira do Serviço Nacional de Saúde. Lisboa: Ministério da Saúde; 2008.

${ }^{25}$. Médici AC, et al. Sistemas de custos como instrumento de eficiência e qualidade dos serviços de saúde. São Paulo: Fundap/IESP; 1995.

${ }^{26}$. Piola SF, Vianna SM. organizadores. Economia da Saúde: conceitos e contribuição para a gestão em saúde. Brasília: Ipea; 1995.

27. Castro JD. A utilização do sistema de custeio por absorção para avaliar custos da atenção básica de saúde: reformulações e aprimoramentos metodológicos. [dissertação]Campinas: Curso de Faculdade de Ciências Médicas, Universidade Estadual de Campinas; 2000.

${ }^{28}$. Silva Junior AG. Modelos tecnoassistenciais em saúde: o debate no campo da saúde coletiva. São Paulo: Hucitec; 1998.

29. Schraiber LB. Políticas públicas e planejamento nas práticas de saúde. Saúde Debate. 1995 47:28-35.

30. Costa L S ; GADELHA, C. A. G. ; BURD, P. ; SANTO, M. . Saúde e Desenvolvimento: Estado da Arte e Desafios. In: X Congresso Brasileiro de Saúde Coletiva - ABRASCO, 2012, Porto Alegre RS. X Congresso Brasileiro de Saúde Coletiva - ABRASCO, 2012.

31. Piola S. Texto de apoio 1391: Saúde no Brasil: algumas questões sobre o sistema único de saúde. Estado de uma nação. Brasília: Ipea; 2009.

32. Conselho Nacional de Secretários Estaduais de Saúde (BR). Coleção para entender a gestão do sus: O financiamento da Saúde. Brasília: Conselho Nacional de Secretários Estaduais de Saúde; 2011.

33. The World Bank. Indicators. Washington: The World Bank; c2015. Disponível em: <http:// data.worldbank.org/indicator/>. Acesso em: 29 out. 2014. 